\title{
Predictors of incubation costs in seabirds: an evolutionary perspective
}

\author{
AKIKO SHOJI, ${ }^{1{ }^{*} \dagger}$ KYLE H. ELLIOTT, ${ }^{2}$ STÉPHANE ARIS-BROSOU,,${ }^{3,4}$ RORY P. WILSON ${ }^{5}$ \& \\ ANTHONY J. GASTON ${ }^{1,3}$ \\ ${ }^{1}$ Environment Canada, National Wildlife Research Centre, Ottawa, ON, $K 1 \mathrm{~A} \mathrm{OH}$, Canada \\ ${ }^{2}$ Department of Zoology, University of Manitoba, Winnipeg, MB, R3T 2N2, Canada \\ ${ }^{3}$ Department of Biology, University of Ottawa, Ottawa, ON, K1N 6N5, Canada \\ ${ }^{4}$ Department of Mathematics and Statistics, University of Ottawa, Ottawa, ON, K1N 6N5, Canada \\ ${ }^{5}$ Biosciences, Swansea University, Swansea SA2 8PP, UK
}

\begin{abstract}
Energy costs during breeding play an important role in the evolution of life history traits. Seabirds show substantial variation in both incubation shift length (ISL) and metabolic rates. However, it is still unclear how variation in life history traits relates to incubation metabolic rates (IMR). Here, we examine the relationship between IMR and life history traits, including ISL, fledging strategy (precocial to altricial), incubation period, nest location (surface vs. underground) and clutch mass relative to adult body mass for 30 species of seabirds collated from the literature. Using both conventional non-phylogenetic and phylogenetic generalized least-squares approaches, we show that IMR is negatively associated with ISL, relative clutch mass and with underground nesting, while fledging strategy and incubation period have no impact on IMR once phylogeny is accounted for. Maximum likelihood reconstructions further suggest than ancestral seabirds had average ISL and relative clutch mass, and were surface nesters. We conclude that lower metabolic rates during incubation are associated with both an increased incubation shift length that allows animals to travel farther, as well as the evolutionary emergence of underground nesting that requires less social interaction.
\end{abstract}

Keywords: ancestral reconstruction, incubation metabolic rate, incubation shift length, life history theory.

Metabolic energy requirements during reproduction are associated with life history traits in animals (McNab 2002, Blomberg et al. 2003, White \& Seymour 2004, Lovegrove 2005). For instance, in productive environments where population density is low compared with food availability, higher levels of energy expenditure - and therefore energy intake are presumed to be selected to sustain a large brood size and intrinsic population growth rate (White \& Seymour 2004). However, what determines these metabolic requirements is still a matter of debate. For instance, accounting for body mass (White

†Present address: Department of Zoology, University of Oxford, Oxford OX1 3PS, UK.

*Corresponding author.

Email: akikosho@gmail.com
2011) still leaves considerable interspecific variation in metabolism unexplained (McKechnie \& Wolf 2004). One possibility is that those animals that regularly encounter long periods of fasting (long fasting endurance) have a lower metabolic rate to increase the probability of survival during the period of fasting (Shibata \& Bukowiecki 1987, Cherel et al. 1988, Overgaard et al. 2002). In birds, incubation shift length (length of time spent incubating eggs between foraging bouts) is one widely reported parameter that varies considerably among orders and provides the possibility of testing for a connection between fasting endurance and metabolic rate.

In birds, reproduction is characterized by periods of potentially high energy costs, in particular during egg laying for females, and periods of fasting during incubation and during the brooding of chicks (Ellis \& Gabrielsen 2002, Elliott et al. 
2013). Although chick rearing is often assumed to be the most energetically expensive part of reproduction (Warham 1990), the energy costs associated with maintaining eggs at a suitable temperature are not negligible (Grant 1981, Niizuma et al. 2005). The metabolic rate at rest can be higher in incubating birds than in chick-rearing birds, partly because higher levels of thyroid hormones increase resting metabolic rate to enable the heating of the eggs (Bech et al. 2002, Rønning et al. 2008, Welcker et al. 2013).

While it is generally recognized that incubation metabolic rate (IMR) is related to basal metabolic rate (Ellis \& Gabrielsen 2002), it is still unclear which life history traits are associated with IMR. A number of previous studies have attempted to explain variation in IMR. Niizuma and Watanuki (1997) suggested that species with longer foraging trips during incubation (and therefore longer incubation shifts) have lower IMR than species with shorter foraging trips because lower IMR facilitates extended fasting needed by the mate that is not foraging. Precocial species, in which offspring are mobile shortly after hatching, usually have relatively larger eggs in proportion to female body mass and longer incubation periods, so that these species are thought to maintain higher IMR than non-precocial (semi-precocial to fully altricial) species (Gloutney et al. 1996), presumably because they transfer more heat from the incubating bird to the egg. Species with long incubation periods often have lower IMR (Thomson et al. 1998, Ellis \& Gabrielsen 2002) to conserve energy for on-duty birds, allowing off-duty birds to stay away longer while foraging. Species that visit their nests during the day may also have higher IMR (Niizuma \& Watanuki 1997). Many burrow or crevice nesters are nocturnal at the colony, only arriving at and departing from their nests at night (Gaston 1992, 2004). Hence opportunities for incubation shift changes are more limited for nocturnal species than for diurnal species, as the duration of the minimum incubation shift is at least $24 \mathrm{~h}$. At the same time, social interactions during incubation are likely to be very restricted (Gaston 1992, 2004), which might cause individuals of nocturnal species to have lower IMR because they may not need to invest in a fully alert brain and muscles at all times. In contrast, open-nesters such as gulls (Laridae) and gannets (Sulidae), which are generally active at the nest in daylight, must be capable of responding quickly to social cues, and are likely to have high IMR because interactions with neighbours are frequent and birds expend time defending both their nests and offspring (Kober \& Gaston 2003). Thus, nest location, either at the surface or underground, may be associated with IMR. Species with larger clutch sizes may have higher energy demands, as the energy required for heating an egg increases with clutch size (Thomson et al. 1998). While links between a number of life history traits and IMR appear plausible (Warham 1990, Ellis \& Gabrielsen 2002), it is still unclear which life history traits best account for broad-scale variation in metabolic rate during incubation as the impact of life history variables on IMR has never been evaluated within a single framework.

To address this question, we conducted an analysis using data from the literature on 30 species of seabirds (Supporting Information Table S1) to assess whether inter-specific variation in incubation shift length affects IMR. We included life history traits that have been reported to affect IMR, as reviewed above and listed in Table S1, including: incubation shift length (ISL: Niizuma \& Watanuki 1997), fledging strategy (FS: Gloutney et al. 1996), incubation period (IP: Thomson et al. 1998), nesting location (NL: Niizuma \& Watanuki 1997 - at the surface or underground) and relative clutch mass (RCM: Grant 1981, Thomson et al. 1998, Tinbergen \& Williams 2002 - relative to adult body mass). In this study, clutch size is equal to either 1 or 2 and thus variation within clutch size is small. However, simply comparing clutch size between species is misleading, because there is inter-specific variation in egg size and energetic demands are not solely determined by clutch size, as energy demands also include producing heat to incubate eggs that are a function of egg size (Ellis \& Gabrielsen 2002). Therefore, we used relative clutch mass, calculated as the product of egg weight $(\mathrm{g})$ and clutch size, divided by adult body mass. We examined the relationship between IMR and these life history traits using a phylogenetic generalized least-squares approach that, uniquely, accounts for phylogenetic uncertainty and indirectly reconstructs ISL at the root of the seabird phylogeny.

\section{METHODS}

We used the definition of seabirds from Schreiber and Burger (2002), who included the orders Sphenisciformes, Procellariiformes, Pelecaniformes and 
Charadriiforms. IMR (J/h), ISL (day: as a proxy for fasting duration), body mass and life history trait data for 30 species of seabirds were compiled from previously published studies (Table S1). In preliminary analyses, both latitude at breeding sites and breeding range for each species were found to have no significant impact on IMR, and were therefore excluded from further analyses. IMR data were obtained by using the Web of Science for all studies that included the terms 'incubation', 'metabol*' and 'energ*' in them. We then examined the references cited in all those studies. Although our list may not be exhaustive (papers not indexed in Web of Science and/or from the non-English literature were most certainly missed), the list of studies extracted should be representative of the body of work published on the subject of IMR. Values of ISL and life history traits were obtained from the same studies in which IMR was collected or from family monographs (Croxall 1982, Warham 1990, Schreiber \& Burger 2002, Brooke 2004). Methods used to estimate IMR in the different studies included analysis of gas respiration, washout rate of doubly labelled water (DLW) and rate of mass loss (Shaffer et al. 2001). Although we used IMR measurements based on different methods, there is a body of literature showing that the results are fairly comparable (Table S1). For example, Speakman (1997) and Speakman and Westerterp (2010) showed that the discrepancy between DLW and respirometry, when averaged across a group, is usually $<5 \%$ and that there was no systematic bias in any direction. Bolton (1996) showed that estimated metabolic rates measured by respirometry and weight loss were similar in European Storm-petrels Hydrobates pelagicus. Thus, we combined the data based on different methods to increase statistical power.

\section{Phylogenetic analyses}

Data were analysed using both conventional analyses (ignoring phylogeny) and in phylogenetically informed analyses. Because the latter analyses require an estimate of branch lengths to account for the amount of genetic change when modelling trait evolution, using a tree from the literature (e.g. Hackett et al. 2008) is not sufficient and estimating a new phylogeny is required. To do so, nucleotide sequences of the species of interest were identified by BLASTN searches (Altschul et al. 1990) and were downloaded from GenBank in
May 2014 for two mitochondrial protein-coding genes: cytochrome oxidase 1 (COXl) and cytochrome $b(\mathrm{CYTb})$. These two genes were chosen because (1) part of COXl is regularly sequenced for the purpose of DNA-barcoding in a large range of organisms (Hajibabaei et al. 2007) and (2) $\mathrm{CYTb}$ is obtained alongside COXl in the context of mitochondrial genome projects. Sequences for 11 additional species were also downloaded to (1) root the phylogenetic tree and (2) stabilize phylogenetic estimation, avoiding the long-branch attraction artefact (Felsenstein 1978) by breaking down long branches as much as possible. Accession numbers are given in Supporting Information Table S2. Each gene was aligned independently with MUSCLE (Edgar 2004), visually inspected and edited with JALVIEW (Clamp et al. 2004); alignments were concatenated manually. The best substitution model, selected with MODELTEST (Akaike information criterion, AIC; Posada \& Crandall 1998), was used to estimate the phylogenetic tree under maximum likelihood with PHYML ver.3.0.1 (Guindon et al. 2010). A combination of NearestNeighbour Interchange and Subtree Pruning and Regrafting heuristics was used to search for the best tree topology. Stability of the phylogenetic estimates was assessed with 1000 bootstrap replicates. The Shimodaira and Hasegawa test (SH test; Shimodaira \& Hasegawa 1999), as implemented in Phylogenetic Analysis by Maximum Likelihood (PAML; Yang 2007), was used to compare alternative phylogenetic reconstructions. Trees were rooted with Rhea americana as an outgroup; both the outgroup and the species added to stabilize phylogenetic estimates were removed for subsequent analyses. Phylogenetic independent contrasts were computed in $\mathrm{R}$ ( $\mathrm{R}$ Development Core Team 2011) with the 'caper' library, using phylogenetic generalized linear models (PGLS; Freckleton et al. 2002). The dependence on the phylogeny of the process governing trait evolution is quantified by a parameter, $\lambda$, with $\lambda=0$ implying complete independence and $\lambda=1$ complete dependence. We therefore further tested the impact of the phylogeny on trait evolution by running the PGLS analysis twice, first by setting $\lambda=1$ and secondly by estimating $\lambda$ by maximum likelihood; we then compared the two models based on AIC for small samples (AICc; Burnham \& Anderson 2002). Because the definition of contrasts depends on the phylogenetic tree (Felsenstein 1985) and because some uncertainty in the reconstruction process 
exists, we computed the $P$-value for each parameter estimates of the PGLS analysis on each of the 1000 bootstrapped trees. This enabled us to define a probability of observing a significant parameter estimate in the face of phylogenetic uncertainty, which we hereafter term the phylogenetic $P$-value, denoted $P_{P}>\alpha$ where $\alpha$ is the significance level.

\section{Statistical analyses}

IMR of all 30 seabird species was described using a general linear model (GLM) with a Gaussian distribution. We checked for normality and homoscedasticity by plotting fitted and observed values and residuals, and we ln-transformed variables when necessary (IMR and ISL). The effect of mass was removed using the residual of regression of IMR on mass for all analysis (see Supporting Information for a statistical justification). In total, we considered 10 candidate models to identify factors (i.e. life history traits, as listed in Table 1) affecting IMR. The model-building procedure employed a multimodel inference approach to test which life history traits best account for broad-scale variation in IMR. We considered all of the models that were biologically reasonable. Models were ranked using AICc. We employed $\triangle \mathrm{AICc}$ and Akaike weights $\left(w_{i}\right)$ to infer support for models in the candidate set (Akaike 1973, Burnham \& Anderson 2002). An AICc-based stepwise model selection procedure was also employed to find the best model. All reported $P$-values are two-tailed, at a significance level $\alpha=0.01$.

To understand the evolutionary implications of the variables detected to affect IMR in seabirds, we performed ancestral reconstructions mapped onto the estimated phylogenetic tree by maximum likelihood (Schluter et al. 1997). Briefly, the evolution of continuous characters was described according to a Brownian model, whereas that of discrete characters was assumed to follow an instantaneous matrix of rate change rate with no constraints placed on individual rates (the 'all rates different' model in APE; Paradis et al. 2004). The likelihood of the ancestral states was computed with a joint estimation procedure (Pupko et al. 2000). As above, phylogenetic uncertainty was accounted for by running these models not only on the estimated tree but also on the set of 1000 bootstrapped trees; the distribution over the bootstrapped trees of the reconstructed state at the root of the seabird tree was then used to predict, from the PGLS modelling, the most likely IMR condition at the origin of seabirds.

\section{RESULTS}

In total, 41 species were aligned for two mitochondrial genes, producing a dataset of 2694 nucleotide sites. The model of evolution selected by AIC was GTR $+\Gamma+I$ (for a review see Aris-Brosou \& Rodrigue 2012). The estimated tree proved relatively robust, as most bootstrap support values were $>0.75$ (Fig. 1). Some of the additional sequences such as Phaethon rubricauda and Haematopus ater were not correctly placed, but as all erroneous parts were caused by the additional sequences, subsequently removed from the tree (see Methods), the results presented below were not affected.

Table 1. Predictions of the hypotheses for assessing which life history trains best account for broad-scale variation in metabolic rate during incubation.

\begin{tabular}{|c|c|c|}
\hline $\begin{array}{l}\text { Related } \\
\text { parameters }\end{array}$ & Definition & Prediction \\
\hline ISL & $\begin{array}{l}\text { Length of time spent incubating eggs between } \\
\text { foraging bouts }\end{array}$ & Species with longer incubation shift length have lower IMR \\
\hline FS & $\begin{array}{l}\text { Offspring developmental status at the } \\
\text { time of fledging }\end{array}$ & $\begin{array}{l}\text { Precocial/semi-precocial species have higher IMR than altricial } \\
\text { species }\end{array}$ \\
\hline IP & $\begin{array}{l}\text { Period from laying an egg(s) to hatching a } \\
\text { chick(s) }\end{array}$ & Incubation period is negatively related to IMR \\
\hline NL & $\begin{array}{l}\text { Nest location related to activity levels while } \\
\text { incubating egg(s) }\end{array}$ & $\begin{array}{l}\text { Open-nesters and therefore diurnal species have higher IMR because } \\
\text { of more social interactions with neighbours and/or predators }\end{array}$ \\
\hline $\mathrm{RCM}$ & Clutch mass as $\%$ adult mass & Larger RCM is positively related to IMR \\
\hline
\end{tabular}

FS, fledging strategy (altricial, semialtricial, semi-precocial or precocial); IP, incubation period (days); ISL, incubation shift length; NL, nest location (surface or underground); RCM, relative clutch mass (clutch mass as \% adult mass). 


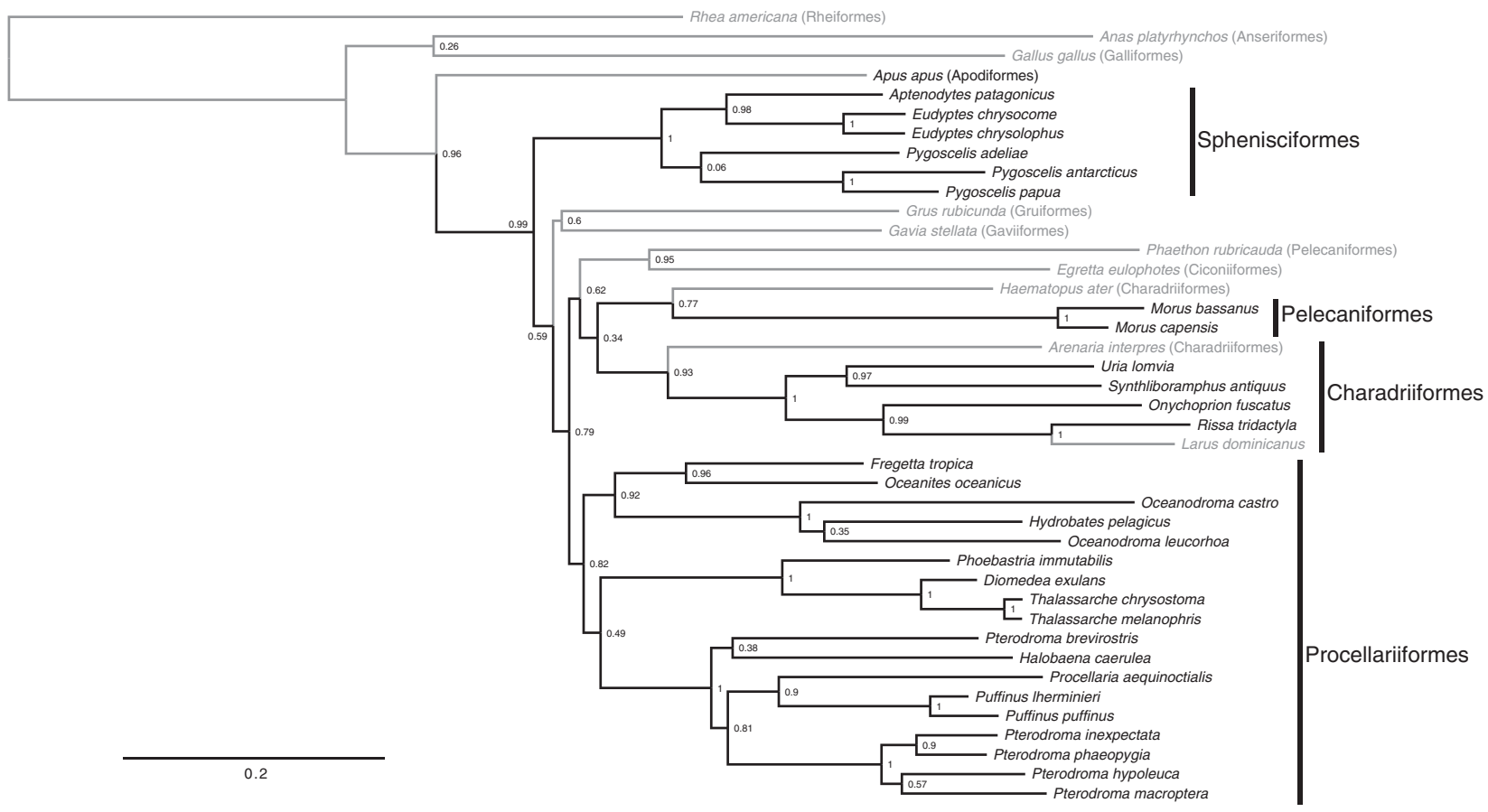

Figure 1. The phylogeny of seabirds. Maximum likelihood tree estimated with PHYML. The species represented in light grey are the additional species included in the phylogenetic analyses, but removed for the PGLS analyses. Scale bar is in expected number of substitutions per sites. Node numbers represent bootstrap values (1000 replicates).

For model selection, we considered the variables presented in Fig. 2: incubation shift length (ISL), fledging strategy (FS), incubation period (IP), nest location (NL) and relative clutch mass (RCM; see Table S1 for details). The regression of ISL on mass-corrected (residual) of $\ln (\mathrm{IMR})$ reduced the null AICc value of 32.82 by $12.15-20.67$ units (Table 2). The effect of fledging strategy was almost as strong as $\ln$ (ISL), as the model including this factor was 24.70-20.67 $=4.03$ AICc units worse than the model with only $\ln ($ ISL). Incubation period on its own increased AICc with respect to the null model (Table 2). However, the best model selected by the stepwise procedure included only $\ln (\mathrm{ISL})+\mathrm{NL}+\mathrm{RCM}$. The standard GLM analysis, which disregards phylogenetic information, shows that all three life history traits, ln(ISL), $\mathrm{NL}$ and RCM, are significant predictors of masscorrected $\ln (\mathrm{IMR})$ (Table 3).

The PGLS analysis, which takes the phylogeny relating the study species into account, showed a significant and negative effect of only $\ln ($ ISL) and RCM (Table 3; $\lambda=1$ ). The same result was obtained when the tree of Hackett et al. (2008) was used, indicating that our results are robust to the relative phylogenetic position of Sphenisciformes and Charadiiformes, which is not surprising, as the two trees are not significantly different (AU test, $P=0.5110$ ). However, the alternative model in which $\lambda$ is estimated by maximum likelihood suggested that these traits evolved independently of the phylogeny (estimated $\lambda=0$ ), as this model had a smaller AICc (Table 3). In this latter case, all three traits (ln(ISL), NL and RCM) were significant. The computation of the phylogenetic $P$-values $P_{P}>\alpha$ confirms that our results are robust to phylogenetic uncertainty, as all $P$-values of the bootstrapped trees are smaller than the significance level except for NL, for which $92 \%$ of the resampled $P$-values satisfied our criterion (Fig. 3).

Although the best model (mass-corrected $\ln (\mathrm{IMR})$ as a function of $\ln (\mathrm{ISL})+\mathrm{NL}+\mathrm{RCM}+\varepsilon$, with $\varepsilon \sim \mathrm{N}(0,1))$ proved to be phylogenetically independent (estimated $\lambda=0$ ), each of the selected variables (ISL, NL and RCM) showed a phylogenetic signal (Fig. $4 \mathrm{a}-\mathrm{C}$ ) with $\lambda$ estimated to be between 0.42 (for ISL) and 1 (for NL and $\mathrm{RCM}$ ). We therefore reconstructed the ancestral states of these three characters on the maximum likelihood tree (Fig. 4d-f) and estimated the state 

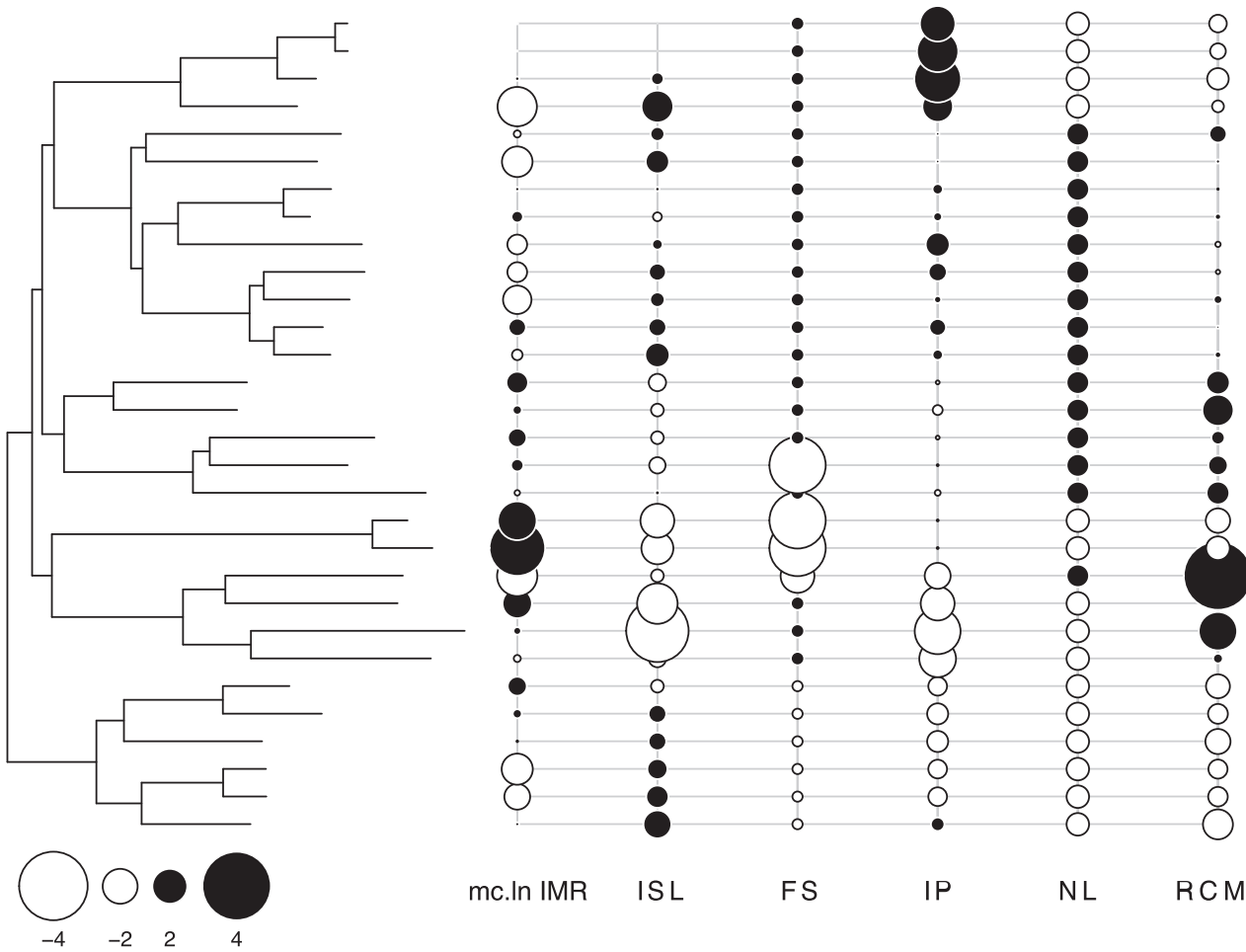

\begin{tabular}{l|l} 
Thalassarche melanophris & \\
Thalassarche chrysostoma & \\
Diomedea exulans & \\
Phoebastria immutabilis & \\
Halobaena caerulea & \\
Pterodroma brevirostris & \\
Puffinus puffinus & \\
Puffinus Iherminieri & \\
Procellaria aequinoctialis & $\mathrm{Pr}$ \\
Pterodroma macroptera & \\
Pterodroma hypoleuca & \\
Pterodroma phaeopygia & \\
Pterodroma inexpectata & \\
Oceanites oceanicus & \\
Fregetta tropica & \\
Oceanodroma leucorhoa & \\
Hydrobates pelagicus & \\
Oceanodroma castro & \\
Morus capensis & \\
Morus bassanus & $\mathrm{Pe}$ \\
Synthliboramphus antiquus & \\
Uria lomvia & \\
Rissa tridactyla & $\mathrm{Ch}$ \\
Onychoprion fuscatus & \\
Pygoscelis papua & \\
Pygoscelis antarcticus & \\
Pygoscelis adeliae & \\
Eudyptes chrysolophus & $\mathrm{Sp}$ \\
Eudyptes chrysocome & \\
Aptenodytes patagonicus &
\end{tabular}

$\begin{array}{llllll}\text { mc.In IMR } & \text { ISL } & \text { FS } & \text { IP } & \text { NL } & \text { RCM }\end{array}$

Figure 2. Summary of the relative distribution of the life history traits in relation to their phylogenetic distribution. The tree on the lefthand side is as in Fig. 1, with the additional species (see Methods) removed. Relative values of each trait are represented on a -4 to 4 scale; discrete traits for NL represent surface (open)/underground (filled) nesters. FS, fledging strategy; IP, incubation period; ISL, In incubation shift length; mc.In IMR, mass-corrected In incubation metabolic rate; NL, nest location; RCM, relative clutch size. See Table S1 for data. Pr, Procellariiformes; Pe, Pelecaniformes; Ch, Charadriiformes; Sp, Sphenisciformes.

Table 2. Summary of standard (phylogenetically unaware) GLM model selection results for the analysis of the determinants of seabird incubation metabolic rates. Ten candidate models as well as the best (stepwise-selected) model were considered, including the null model (intercept only).

\begin{tabular}{lccccc}
\hline Statistical model & AICc & AAICc & $w_{i}$ & Deviance & $K$ \\
\hline ISL + NL + RCM & 2.76 & 0.0 & 0.88 & 1.38 & 4 \\
ISL + FS + NL + & 7.56 & 4.8 & 0.08 & 1.24 & 6 \\
IP + RCM & & & & & \\
ISL + FS + NL + IP & 9.78 & 7.02 & 0.03 & 1.43 & 5 \\
ISL + FS & 11.99 & 9.23 & 0.01 & 1.76 & 3 \\
ISL + FS + NL & 13.76 & 11.00 & 0 & 1.74 & 4 \\
ISL & 20.67 & 17.92 & 0 & 2.86 & 2 \\
FS & 24.7 & 21.94 & 0 & 2.87 & 2 \\
RCM & 33.13 & 30.37 & 0 & 4.34 & 2 \\
NL & 33.61 & 30.85 & 0 & 4.41 & 2 \\
IP & 34.49 & 31.73 & 0 & 4.54 & 2 \\
Null & 32.82 & 30.06 & 0 & 4.59 & 1 \\
\hline
\end{tabular}

AICc, Akaike's information criterion for small samples; $\triangle \mathrm{AICc}$, the change in AICc relative to the best (minimum AICc) model; $w_{\mathrm{i}}$, Akaike weight; $K$, number of parameters. The residual deviance is twice the difference in log-likelihood between each model and the full model. See notes to Table 1 for other abbreviations. IP and ISL were In-transformed. at the root of the seabird tree for ISL, NL and RCM over the set of 1000 bootstrapped trees (Fig. 4g-i, respectively). The mode of these maximum likelihood reconstructions shows that ancestral seabirds had a most likely $\ln$ (ISL) of 1.58 , was a surface nester and had an RCM of $13.3 \%$ (Fig. 4). Predicted values of these trait-specific PGLS analyses show that both ancestral ISL and RCM were almost identical to the mean value observed today (paired $t$-test; ISL: $t=0.13$, $\mathrm{df}=29, \quad P=0.8951 ; \quad \mathrm{RCM}: \quad t=0.34, \quad \mathrm{df}=29$, $P=0.7342$; Supporting Information Fig. S1). Taken together, these ancestral reconstructions suggest a mass-corrected $\ln (\mathrm{IMR})$ of ancestral seabirds of between 0.023 and 0.030 (Fig. 4, Supporting Information Fig. S2), which is close to what is found today in the genus Thalassarche (albatrosses, Procellariiformes).

\section{DISCUSSION}

We set out to investigate the relationship between IMR and a range of life history traits in free-ranging 
Table 3. Summary table of the best AICc model run under the standard GLM and under PGLS, either fixing $\lambda$ to 1 or estimating it by maximum likelihood.

\begin{tabular}{|c|c|c|c|c|c|c|c|c|c|}
\hline & \multicolumn{3}{|c|}{ GLM } & \multicolumn{3}{|c|}{ PGLS $\lambda=1$} & \multicolumn{3}{|c|}{ PGLS $\lambda$ estimated } \\
\hline & Est. & se & $P$ & Est. & se & $P$ & Est. & se & $P$ \\
\hline ISL & -0.43 & 0.06 & $6.7 \times 10^{-8}$ & -0.36 & 0.09 & 0.0006 & -0.43 & 0.06 & $7.3 \times 10^{-8}$ \\
\hline NL & 0.38 & 0.12 & 0.0047 & 0.33 & 0.32 & 0.3076 & 0.39 & 0.13 & 0.0048 \\
\hline $\mathrm{RCM}$ & -0.04 & 0.01 & $2.0 \times 10^{-5}$ & -0.03 & -1.14 & 0.0229 & -0.04 & 0.01 & $4.0 \times 10^{-5}$ \\
\hline
\end{tabular}

All three models fitted $\ln (\mathrm{ISL})+\mathrm{NL}+\mathrm{RCM}+\varepsilon$, with $\varepsilon$ being a mean-zero normally distributed error term, to mass-corrected $\ln (\mathrm{IMR})$. AICc values under PGLS are 18.79 when $\lambda$ is set to 1 and 2.68 when $\lambda$ is estimated; the estimated $\lambda$ is 0 . ISL was In-transformed. Est., parameter estimate; se, standard error.

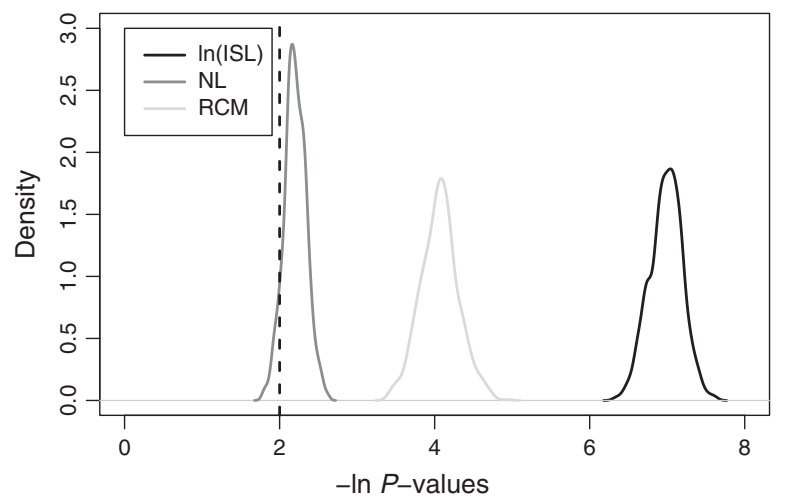

Figure 3. Distributions of negative In $P$-values under PGLS accounting for phylogenetic uncertainty. PGLS densities of $P$ values computed for each of the 1000 bootstrapped trees are plotted for In(ISL) (black line), NL (dark grey line) and RCM (light grey line). The vertical broken line represents the significance level adopted here $(\alpha=0.01)$.

seabirds (Fig. 2). This approach, based on the computation of phylogenetically aware $P$-values $P_{P}>\alpha$, confirms that IMR is not associated with fledging strategy or incubation period, but is associated with ISL, NL and RCM (Table 3). This result supports previous studies indicating that ISL, NL (Niizuma \& Watanuki 1997) and RCM (Grant 1981, Thomson et al. 1998, Tinbergen \& Williams 2002) are the main factors influencing IMR in seabirds. Hence, constraints associated with foraging far from the colony seem to be a major factor influencing life history strategies in marine birds (Lack 1968, Gaston 2004). Indeed, one of the most important consequences of distant foraging is the need for prolonged foraging trips. Because both partners alternate incubation, the resulting prolonged fasting by the incubating bird may cause selection for reduced IMR to minimize energy expenditure during long incubation shifts.
Although we found that the ancestral seabird had an average ISL relative to values observed in extant seabirds, the need to reduce energy expenditure during incubation may explain why some species, such as the Ancient Murrelet Synthliboramphus antiquus, which have low IMR, line their nest with insulating material to increase heat efficiency (Gaston \& Jones 1998). Our result raises the question of why birds with short ISL retain elevated IMR. Presumably, there are tradeoffs associated with lower IMR, such as lower egg temperature and slower embryo development, or difficulty switching to higher levels of metabolism during at-sea active periods. The importance of various physiological tradeoffs remains to be fully elucidated.

RCM, also selected in the best model, was found to be negatively related to IMR, contrary to our prediction that as the demands of incubation increase with RCM, IMR should increase with clutch size (Thomson et al. 1998). However, species with large RCM in this study included Ancient Murrelets and Storm-petrels (Fig. 2), both of which have relatively long ISL and elevated predation risk on arrival at the colony (Warham 1990, Gaston 1992). Thus, the relatively low IMR in those species, relative to their RCM, may be due to reduced energy expenditure during incubation, so that these birds can extend foraging trip duration (and therefore the partner's incubation duty) on nights when predation risk is high, for example during moon-lit nights (Shoji et al. 2011) or when weather is inclement during the brief period available to switch-over.

A little-explored factor potentially affecting IMR is variation in the timing of nest visitations, which is highly correlated with nest location (NL: surface vs. underground). We found NL has a 

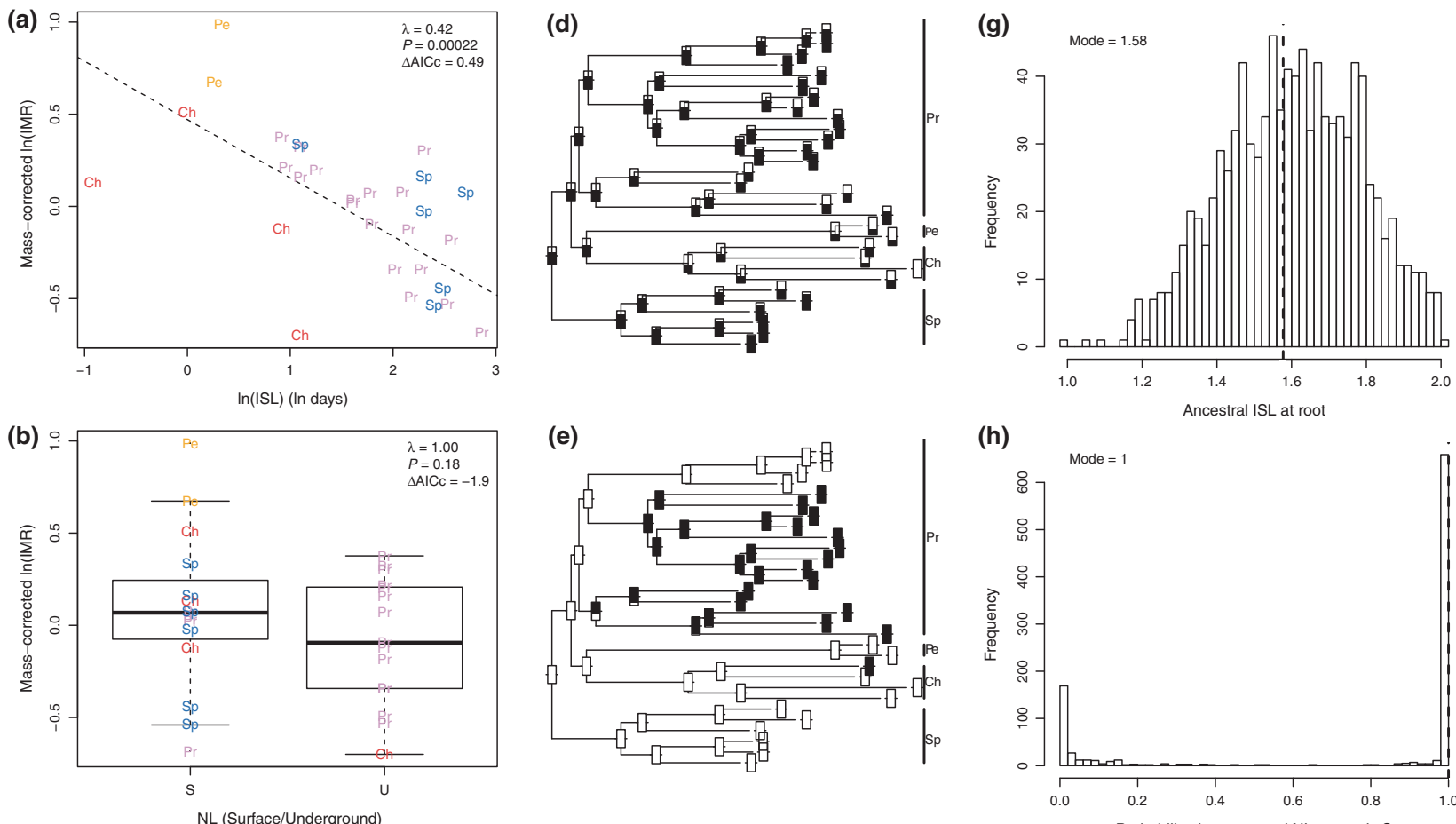

(h)
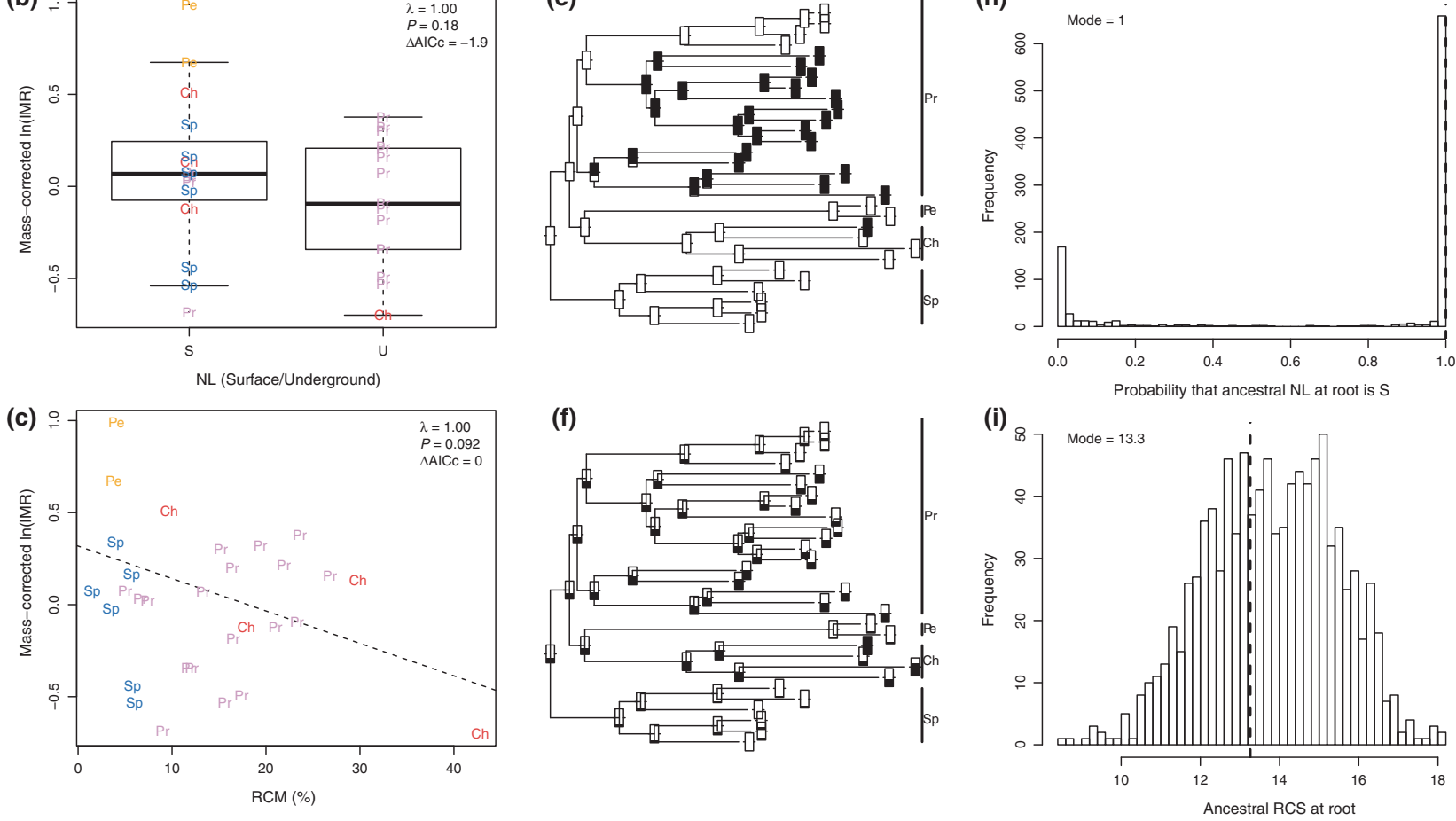

Figure 4. Ancestral reconstructions of individual significant traits identified in the joint PGLS analyses: In(ISL), NL and RCM. (a-c) Individual PGLS analyses; shown in top right corners: estimates of $\lambda$, significance of the PGLS analysis with $\lambda$ estimated as a free parameter, $\triangle \mathrm{AIC}$ between this model and the one constraining $\lambda$ to be zero. (d-f) Maximum likelihood mapping of ancestral states on the phylogeny; relative (on a 0-1 scale) values are represented as thermometers for continuous traits (In(ISL) and RCM); for NL, a discrete trait, open rectangles represent surface nesting. Clades are shown as vertical bars: Pr, Procellariiformes; Pe, Pelecaniformes; Ch, Charadriiformes; Sp, Sphenisciformes. (g-i) Ancestral reconstructions of the root state for In(ISL), NL and RCM over the 1000 bootstrapped trees. Model values are shown as vertical broken lines; actual values shown in top left corners.

strong effect on IMR in addition to ISL and RCM (Table 2). The difference in IMR between species may be related to activity patterns because open-nesters such as Sooty Terns Onychoprion fuscatus exhibit higher activity levels (e.g. defending their mate and eggs). This, in retrospect, is not unexpected because nocturnal species are normally in a small underground burrow sitting on their egg for a day or up to several weeks focusing on incubation, whereas diurnal species (surface nesters) face continuous interactions with neighbours and predators, necessitating a higher IMR.

In conclusion, our analysis of the relationship between seabird IMR and life history traits revealed a significant association between IMR and ISL, relative clutch size and nest location, but no association with incubation period or fledging 
strategy. Our results further suggest that the ancestral seabird was a surface nester with average ISL and RCM in terms of today's values, so that lower metabolic rates during incubation are most likely associated with the evolutionary emergence of underground nesting. A deeper understanding of the evolution of life history traits and, critically, of their tradeoffs will require more data from additional species.

We would like to thank three anonymous referees as well as the Editor and Associate Editor for comments that helped improve this paper. This work was funded by Environment Canada (A.S., A.J.G.), Japan Student Services Organization, Merton College (A.S.), the Natural Sciences and Engineering Research Council of Canada (S.A.B., K.H.E.) and by the Canada Foundation for Innovation (S.A.B.).

\section{REFERENCES}

Akaike, H. 1973. Information theory as an extension of the maximum likelihood principle. In Petrov, B.N. \& Csaki, F. (eds) Second International Symposium on Information Theory: 267-281. Budapest: Akademiai Kiado.

Altschul, S.F., Gish, W., Miller, W., Myers, E.W. \& Lipman, D.J. 1990. Basic local alignment search tool. J. Mol. Biol. 215: 403-410.

Aris-Brosou, S. \& Rodrigue, N. 2012. The essentials of computational molecular evolution. Methods Mol. Biol. 855: 111-152.

Bech, C., Langseth, I., Moe, B., Fyhn, M. \& Gabrielsen, G.W. 2002. The energy economy of the arctic-breeding Kittiwake (Rissa tridactyla): a review. Comp. Biochem. Physiol. A Mol. Integr. Physiol. 133: 765-770.

Blomberg, S.P., Garland, T. \& Ives, A.R. 2003. Testing for phylogenetic signal in comparative data: behavioral traits are more labile. Evolution 57: 717-745.

Bolton, M. 1996. Energy expenditure, body-weight and foraging performance of Storm Petrels Hydrobates pelagicus breeding in artificial nesting chambers. Ibis 138 : 405-409.

Brooke, M.D.L. 2004. Albatrosses and Petrels across the World: Procellariiformes (Bird Families of the World). Oxford: Oxford University Press.

Burnham, K.P. \& Anderson, D.R. 2002. Model Selection and Multimodel Inference: A Practical Information-theoretic Approach. New York: Springer.

Cherel, Y., Robin, J.P., Walch, O., Karmann, H., Netchitailo, P. \& Lemaho, Y. 1988. Fasting in King Penguin. I. Hormonal and metabolic changes during breeding. Am. J. Physiol. 254: R170-R177.

Clamp, M., Cuff, J., Searle, S.M. \& Barton, G.J. 2004. The Jalview Java alignment editor. Bioinformatics 20: 426-427.

Croxall, J.P. 1982. Energy costs of incubation and moult in petrels and penguins. J. Anim. Ecol. 51: 177-194.

Edgar, R.C. 2004. MUSCLE: multiple sequence alignment with high accuracy and high throughput. Nucleic Acids Res 32: 1792-1797.
Elliott, K.H., Ricklefs, R.E., Gaston, A.J., Hatch, S.A., Speakman, J.R. \& Davoren, G.K. 2013. High flight costs, but low dive costs, in auks support the biomechanical hypothesis for flightlessness in penguins. Proc. Natl Acad. Sci. USA 110: 9380-9384.

Ellis, H.I. \& Gabrielsen, G.W. 2002. Energetics of freeranging seabirds. In Schreiber, E.A. \& Burger, J. (eds) Biology of Marine Birds: 359-407. Boca Raton: CRC Press.

Felsenstein, J. 1978. Cases in which parsimony or compatibility methods will be positively misleading. System. Zool. 27: 401-410.

Felsenstein, J. 1985. Phylogenies and the comparative method. Am. Naturalist 125: 1-15.

Freckleton, R.P., Harvey, P.H. \& Pagel, M. 2002. Phylogenetic analysis and comparative data: a test and review of evidence. Am. Naturalist 160: 712-726.

Gaston, A.J. 1992. The Ancient Murrelet: A Natural History in the Queen Charlotte Islands. London: T \& AD Poyser.

Gaston, A.J. 2004. Seabirds: A Natural History. New Haven: Yale University Press.

Gaston, A.J. \& Jones, I.L. 1998. The Auks: Alcidae. Oxford: Oxford University Press.

Gloutney, M.L., West, N. \& Clark, R.G. 1996. Metabolic costs of incubation and clutch size determination in the Red Junglefowl, Gallus gallus spadiceus. Comp. Biochem. Physiol. A Physiol. 114: 265-270.

Grant, G. 1981. Energy cost of incubation to the parent seabird. In Whittow, G.C. \& Rahn, H. (eds) Seabird Energetics. New York: Plenum Press.

Guindon, S., Dufayard, J.F., Lefort, V., Anisimova, M., Hordijk, W. \& Gascuel, O. 2010. New algorithms and methods to estimate maximum-likelihood phylogenies: assessing the performance of PhyML 3.0. System. Biol. 59: 307-321.

Hackett, S.J., Kimball, R.T., Reddy, S., Bowie, R.C.K., Braun, E.L., Braun, M.J., Chojnowski, J.L., Cox, W.A., Han, K.L., Harshman, J., Huddleston, C.J., Marks, B.D., Miglia, K.J., Moore, W.S., Sheldon, F.H., Steadman, D.W., Witt, C.C. \& Yuri, T. 2008. A phylogenomic study of birds reveals their evolutionary history. Science 320: 1763-1768.

Hajibabaei, M., Singer, G.A.C., Hebert, P.D.N. \& Hickey, D.A. 2007. DNA barcoding: how it complements taxonomy, molecular phylogenetics and population genetics. Trends Genet. 23: 167-172.

Kober, K. \& Gaston, A.J. 2003. Social interactions among breeding Brunnich's Guillemots Uria lomvia suggest constraints in relation to offspring vulnerability. Ibis 145 : 413-418.

Lack, D.L. 1968. Ecological Adaptations for Breeding in Birds. London: Methuen.

Lovegrove, B.G. 2005. Seasonal thermoregulatory responses in mammals. J. Comp. Physiol. B 175: 231-247.

McKechnie, A.E. \& Wolf, B.O. 2004. Partitioning of evaporative water loss in White-winged Doves: plasticity in response to short-term thermal acclimation. J. Exp. Biol. 207: 203-210.

McNab, B.K. 2002. Physiological Ecology of Vertebrates. Ithaca, NY: Comstock Cornell.

Niizuma, Y. \& Watanuki, Y. 1997. Effects of circadian rhythm and breeding stage on resting metabolic rates in fasting Leach's Storm-Petrels. J. Yamashina Inst. Ornithol. 29: 8390. 
Niizuma, Y., Takagi, M., Senda, M., Chochi, M. \& Watanuki, Y. 2005. Incubation capacity limits maximum clutch size in Black-tailed Gulls Larus crassirostris. J. Avian Biol. 36: 421427.

Overgaard, J., Andersen, J.B. \& Wang, T. 2002. The effects of fasting duration on the metabolic response to feeding in Python molurus: an evaluation of the energetic costs associated with gastrointestinal growth and upregulation. Physiol. Biochem. Zool. 75: 360-368.

Paradis, E., Claude, J. \& Strimmer, K. 2004. APE: analyses of phylogenetics and evolution in R language. Bioinformatics 20: 289-290.

Posada, D. \& Crandall, K.A. 1998. Modeltest: testing the model of DNA substitution. Bioinformatics 14: 817-818.

Pupko, T., Pe'er, I., Shamir, R. \& Graur, D. 2000. A fast algorithm for joint reconstruction of ancestral amino acid sequences. Mol. Biol. Evol. 17: 890-896.

R Development Core Team. 2011. R: A Language and Environment for Statistical Computing. Vienna: $\mathrm{R}$ Foundation for Statistical Computing.

Rønning, B., Moe, B., Chastel, O., Broggi, J., Langset, M. \& Bech, C. 2008. Metabolic adjustments in breeding female Kittiwakes (Rissa tridactyla) include changes in kidney metabolic intensity. J. Comp. Physiol. B 178: 779-784.

Schluter, D., Price, T., Mooers, A.O. \& Ludwig, D. 1997. Likelihood of ancestor states in adaptive radiation. Evolution 51: 1699-1711.

Schreiber, E.A. \& Burger, J. 2002. Biology of Marine Birds. New York: CRC Press.

Shaffer, S.A., Costa, D.P. \& Weimerskirch, H. 2001. Comparison of methods for evaluating energy expenditure of incubating wandering albatrosses. Physiol. Biochem. Zool. 74: 823-831.

Shibata, H. \& Bukowiecki, L.J. 1987. Regulatory alterations of daily energy expenditure induced by fasting or overfeeding in unrestrained rats. J. Appl. Physiol. 63: 465470.

Shimodaira, H. \& Hasegawa, M. 1999. Multiple comparisons of log-likelihoods with applications to phylogenetic inference. Mol. Biol. Evol. 16: 1114-1116.

Shoji, A., Elliott, K.H., Aris-Brosou, S., Crump, D. \& Gaston, A.J. 2011. Incubation patterns in a central-place forager affect lifetime reproductive success: scaling of patterns from a foraging bout to a lifetime. PLoS One 6: e17760.

Speakman, J.R. 1997. Doubly Labelled Water - Theory and Practice. London: Chapman and Hall.

Speakman, J.R. \& Westerterp, K.R. 2010. Reverse epidemiology, obesity and mortality in chronic kidney disease: modelling mortality expectations using energetics. Blood Purif. 29: 150-157.

Thomson, D.L., Monaghan, P. \& Furness, R.W. 1998. The demands of incubation and avian clutch size. Biol. Rev. Cambridge Philos. Soc. 73: 293-304.

Tinbergen, J.M. \& Williams, J.B. 2002. Energetics of incubation. In Deeming, D.C. (ed.) Avian Incubation Behaviour, Environment, and Evolution: 299-313. New York: Oxford University Press.

Warham, J. 1990. The Petrels: Their Ecology and Breeding Systems. London: Academic Press.

Welcker, J., Chastel, O., Gabrielsen, G.W., Guillaumin, J., Kitaysky, A.S., Speakman, J.R., Tremblay, Y. \& Bech, C. 2013. Thyroid hormones correlate with basal metabolic rate but not field metabolic rate in a wild bird species. PLOS One 8: e56229.

White, C.R. 2011. Allometric estimation of metabolic rates in animals. Comp. Biochem. Physiol. A Mol. Integr. Physiol. 158: $346-357$.

White, C.R. \& Seymour, R.S. 2004. Does basal metabolic rate contain a useful signal? Mammalian BMR allometry and correlations with a selection of physiological, ecological, and life-history variables. Physiol. Biochem. Zool. 77: 929-941.

Yang, Z. 2007. PAML 4: phylogenetic analysis by maximum likelihood. Mol. Biol. Evol. 24: 1586-1591.

Received 5 September 2013; revision accepted 4 October 2014 Associate Editor: Lorien Pichegru.

\section{SUPPORTING INFORMATION}

Additional Supporting Information may be found in the online version of this article:

Table S1. Data used in this study, including adult body mass $(\mathrm{M}, \mathrm{g})$, incubation metabolic rate (IMR, J g-1 h-1), calculated/measured.

Table S2. Accession numbers of the sequences used in this study.

Figure S1. Distibution of observed ISL (A) and RCS (B) and their estimated ancestral values.

Figure S2. Ancestral reconstructions of individual significant traits identified in the joint PGLS analyses: ISL, NL and RCS. 